\title{
ChemComm
}

\section{Negative area compressibility in silver(I) tricyanomethanide $\uparrow$}

Cite this: Chem. Commun., 2014 50,5264

Received 13th September 2013, Accepted 9th December 2013

DOI: $10.1039 / c 3 c c 47032 f$

www.rsc.org/chemcomm

\author{
Sarah A. Hodgson, ${ }^{a}$ Jasper Adamson, ${ }^{a}$ Sarah J. Hunt, ${ }^{a}$ Matthew J. Cliffe, ${ }^{a}$ \\ Andrew B. Cairns, ${ }^{a}$ Amber L. Thompson, ${ }^{a}$ Matthew G. Tucker, ${ }^{\text {bc }}$ \\ Nicholas P. Funnell ${ }^{a}$ and Andrew L. Goodwin ${ }^{*^{a}}$
}

The molecular framework $\mathrm{Ag}(\mathrm{tcm})\left(\mathrm{tcm}^{-}=\right.$tricyanomethanide) expands continuously in two orthogonal directions under hydrostatic compression. The first of its kind, this negative area compressibility behaviour arises from the flattening of honeycomb-like layers during rapid pressure-driven collapse of the interlayer separation.

In the absence of a structural phase transition, it is a thermodynamic requirement that a system reduce its volume under hydrostatic pressure. ${ }^{1}$ The phenomenon of negative compressibility is the counterintuitive effect whereby this volume reduction couples to an expansion of the material in at least one linear dimension. ${ }^{2}$ Examples of such behaviour are rare even amongst anisotropic materials because buckling and bond compression mechanisms are almost always more effective at reducing crystal volume than is uniaxial or biaxial lattice expansion. Not only does the existence of negative compressibility challenge our understanding of the ways in which materials can respond to external stimuli, but the phenomenon is also of practical importance. It promises a means of developing artificial muscles, nanoscale actuators and high-performance pressure sensors for sonar and altitude measurements. ${ }^{2,3}$ In principle, the most extreme such response allowed thermodynamically is that of negative area compressibility (NAC), whereby a material expands along two orthogonal directions on increasing hydrostatic pressure. The possibility that tetragonal $\mathrm{PbTiO}_{3}$ might show an extremely weak NAC effect has been raised ${ }^{4}$ and disputed $;{ }^{5}$ similarly weak effects are thought to occur at high pressure in $\mathrm{NaV}_{2} \mathrm{O}_{5}$ (ref. 6) and are predicted for $\mathrm{TlGaSe}_{2}{ }^{7}$

\footnotetext{
${ }^{a}$ Department of Chemistry, University of Oxford, Inorganic Chemistry Laboratory, South Parks Road, Oxford OX1 3QR, UK. E-mail: andrew.goodwin@chem.ox.ac.uk; Fax: +44 (0)1865 274690; Tel: +44 (0)1865 272137

${ }^{b}$ ISIS Facility, Rutherford Appleton Laboratory, Chilton, Didcot, OX11 OQX, UK ${ }^{c}$ Diamond Light Source, Harwell Campus, Didcot, OX11 ODE, UK

$\dagger$ Electronic supplementary information (ESI) available: Synthesis; experimental methods; X-ray single-crystal diffraction refinement details (variable-temperature and variable-pressure); neutron powder diffraction refinement details; calculations. CCDC 961474-961482. For ESI and crystallographic data in CIF or other electronic format see DOI: 10.1039/c3cc47032f
}

Instead the remarkably few negative compressibility materials that have been characterised unambiguously expand only along one principal axis when compressed hydrostatically (i.e., negative linear compressibility, NLC). ${ }^{8-12}$ The distinction between NLC and NAC is more fundamental than magnitude alone: NAC materials increase their surface area under hydrostatic pressure, and so they can be used as substrates to provide order-ofmagnitude amplification of piezoelectric response in e.g. ferroelectric sensors. ${ }^{2}$

One strategy for identifying negative compressibility candidates is to target framework materials that exhibit anisotropic negative thermal expansion (NTE): a material that shrinks in one direction on heating is likely to expand in that same direction on compression. ${ }^{13}$ While there is no requirement that deformation mechanisms under temperature and pressure be identical, ${ }^{14}$ there is empirical evidence of such a correspondence amongst molecular framework materials. ${ }^{8,9,12,15}$ The natural strategy then for identifying NAC candidates is to investigate framework materials that exhibit area-NTE. It is for this reason that we chose to investigate silver(I) tricyanomethanide, $\mathrm{Ag}(\mathrm{tcm})$ : its layer-like network topology [Fig. 1(a)] is of a form often associated with area-NTE. ${ }^{16,17}$ In this compound, each $\mathrm{Ag}^{+}$ cation is coordinated by three tcm ${ }^{-}$anions in an approximately trigonal arrangement; likewise each tcm ${ }^{-}$anion is coordinated by three $\mathrm{Ag}^{+}$centres. $^{18,19}$ The resulting hexagonal $(6,3)$ topology is sufficiently open that two honeycomb networks interpenetrate within each layer of the crystal structure [Fig. 1(b)]. Adjacent layers interact via long $\mathrm{Ag} \cdots \mathrm{N}$ contacts $[d(\mathrm{Ag} \cdots \mathrm{N})>3 \AA]$ that are sufficiently weak that the material behaves essentially as a two-dimensional framework, capable even of intercalation chemistry. ${ }^{19}$

Using single-crystal X-ray diffraction, we first established the thermal expansion behaviour of $\mathrm{Ag}(\mathrm{tcm})$ to verify whether or not the material exhibits area-NTE. Because the material crystallises in the orthorhombic (polar) space group Ima2 ${ }^{18}$ its thermal expansivity is uniquely determined by the relative rate of change of the unit cell lengths with temperature. ${ }^{17}$ We observe essentially linear thermal variation in these parameters over the 
(a)

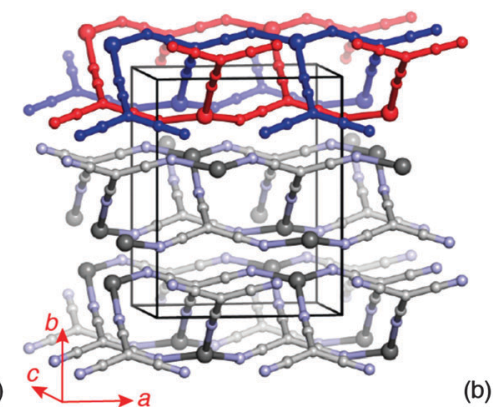

(b)

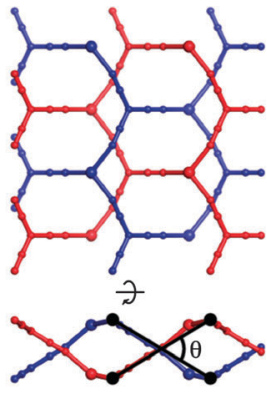

(c) $\nleftarrow$

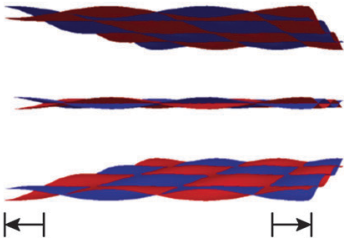

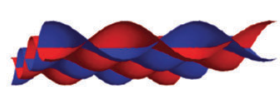

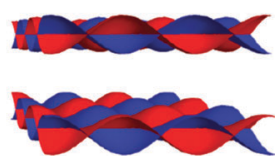

Fig. 1 (a) The crystal structure of $\mathrm{Ag}(\mathrm{tcm})$ is composed of stacked doubly-interpenetrating (6,3)-nets in which both $\mathrm{Ag}^{+}$(large spheres) and $\mathrm{tcm}^{-}$ions (smaller spheres) function as trigonal nodes. (b) The extent of rippling of layers (viewed along $b$ and $a$ in the top and bottom parts of this panel, respectively) can be quantified by the inter-network angle $\theta$ (see ESI $\dagger$ ). (c) The generic mechanical response of a layered material links volume expansion to increasing interlayer separation and increasing layer rippling. The cross-sectional area of each layer is maximised by reducing $\theta$, and hence increases during volume reduction under hydrostatic compression.

temperature range 100-250 K [Fig. 2(a)], yielding coefficients of thermal expansion of $-48(3),+200(2)$ and $-54.0(4) \mathrm{MK}^{-1}$ along the $a, b$, and $c$ axes, respectively. ${ }^{17}$ As is increasingly found to be the case for molecular frameworks, ${ }^{13,20}$ the magnitudes of these values are large compared to those obtained for traditional engineering materials, for which one expects linear coefficients of thermal expansion in the vicinity of $+20 \mathrm{MK}^{-1}$ (i.e., a $0.2 \%$ increase in linear dimension for each $100 \mathrm{~K}$ temperature rise). ${ }^{21}$ What is clear is that $\mathrm{Ag}(\mathrm{tcm})$ does exhibit area-NTE, with an area coefficient of thermal expansion for the $(a, c)$-plane, $\alpha_{(010)}=$ $-102(3) \mathrm{MK}^{-1}$, that is the most extreme negative value reported for a layered material. The material undergoes a $1 \%$ reduction in area for each $100 \mathrm{~K}$ rise in temperature-more than an order of magnitude larger than the corresponding values for area-NTE systems such as graphite and $\mathrm{Ni}(\mathrm{CN})_{2}\left(\alpha_{A}=-2.4\right.$ and $-13 \mathrm{MK}^{-1}$, respectively). ${ }^{17,22,23}$

The microscopic mechanism responsible for this behaviour can be deduced from the variation in framework geometry during heating. The established model of area-NTE in layered materials predicts coupling between NTE and the extent of layer 'rippling', as quantified by the inter-network torsion angle $\theta$ [Fig. 1(b and c)]. ${ }^{16}$ Our measurements indeed indicate an increase in $\theta$ with temperature [Fig. 2(b)]. ${ }^{24}$ Using simple geometric arguments, the temperature dependence of $\theta$ can be used to estimate the value of $\alpha_{A}$ attributable to layer rippling:

$$
\alpha_{A}^{\text {calc }}=-\theta \tan \left(\frac{\theta}{2}\right) \alpha_{\theta},
$$

where $\alpha_{\theta}=\mathrm{d}(\ln \theta) / \mathrm{d} T$ (see $\mathrm{ESI} \dagger$ for derivation). Our data give $\alpha_{A}^{\text {calc }}=-101 \mathrm{MK}^{-1}$, which is within experimental error of the

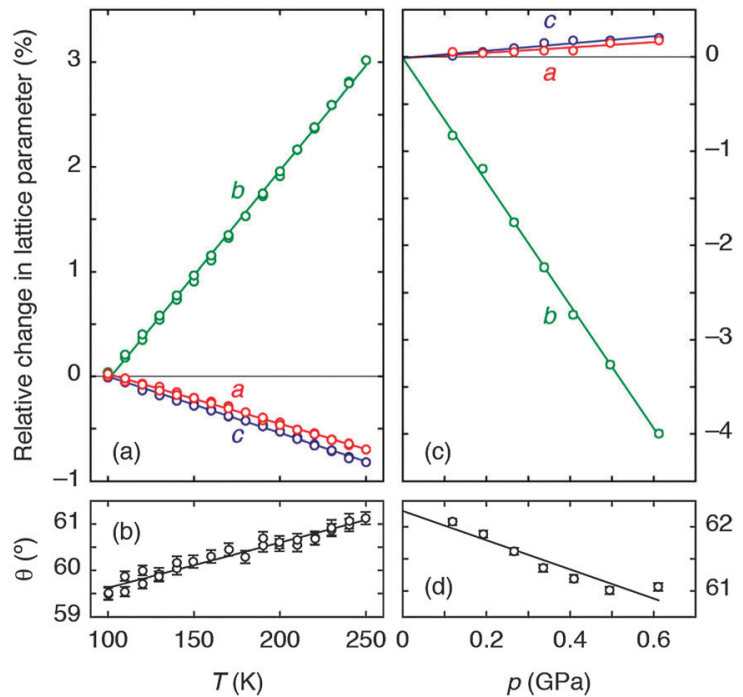

Fig. 2 (a) The thermal variation of lattice parameters, determined using single-crystal X-ray diffraction measurements, is illustrated here for ease of comparison as a \% change relative to the extrapolated value at $100 \mathrm{~K}$. Solid lines are weighted linear fits to the data obtained using the program PASCal. ${ }^{17}$ (b) The corresponding variation in inter-network angle $\theta$ indicates an increase in layer rippling on heating. (c) The pressure dependence of lattice parameters, measured using variable-pressure neutron diffraction, is represented as a \% change relative to the extrapolated value at $0 \mathrm{GPa}$. (d) The corresponding variation in $\theta$ now indicates a decrease in layer rippling under compression.

observed value of $-102(3) \mathrm{MK}^{-1}$. Consequently, we can be confident that the mechanism illustrated in Fig. 1(c) is responsible for area-NTE in $\mathrm{Ag}(\mathrm{tcm})$.

In order to determine whether or not $\mathrm{Ag}(\mathrm{tcm})$ exhibits the target property of NAC, we performed a series of neutron diffraction measurements across the pressure range $0<p<2$ GPa using the PEARL instrument at ISIS. The use of neutron radiation maximises the scattering sensitivity to $\mathrm{C} / \mathrm{N}$ atoms in the presence of $\mathrm{Ag}$ atoms, which is all the more important for measurements performed in the high-background sample environments required for variable-pressure experiments. We found that, for powdered samples, the ambient phase is stable up to $0.615(6) \mathrm{GPa}$, but by $0.672(7) \mathrm{GPa}$ it has transformed reversibly to a related phase with symmetry not higher than monoclinic. The same transformation occurs at higher pressures $\left(0.83<p_{\mathrm{c}}<1.47 \mathrm{GPa}\right)$ in single-crystal experiments (see $\mathrm{ESI} \dagger$ for further discussion); our focus here is on the pressure-dependent behaviour of the ambient phase rather than the nature of the phase transition or the high-pressure phase. Rietveld refinement against the corresponding neutron diffraction patterns revealed the anticipated increase in $a$ and $c$ parameters [Fig. 2(c)], with the anomalous expansion of the material in these directions being allowed by a much more rapid decrease in the interlayer spacing (given by the lattice parameter $b$ ). Our variable-pressure lattice parameter data can be converted into compressibilities using the relationship $K_{\ell}=-[\partial(\ln \ell) / \partial p]_{T}$, from which we obtain $K_{a}=-3.5(6) \mathrm{TPa}^{-1}, K_{b}=+66(20) \mathrm{TPa}^{-1}$, and $K_{c}=-4.0(6) \mathrm{TPa}^{-1}$. Consequently, the compressibility is negative everywhere within the $(a, c)$-plane, with the magnitude 
of NAC quantified by the corresponding area compressibility $K_{(010)}=K_{a}+K_{c}=-7.5(8) \mathrm{TPa}^{-1}$.

Conventional engineering materials (e.g. iron) have linear compressibilities $K \sim 5 \mathrm{TPa}^{-1}$ that correspond to a decrease of ca. $0.5 \%$ in length for each $1 \mathrm{GPa}$ pressure increase; ${ }^{25}$ consequently, the NAC behaviour of $\mathrm{Ag}(\mathrm{tcm})$ is essentially as strong as the positive area compressibility of these 'normal' phases. Moreover, despite the fact that the orthorhombic symmetry of $\mathrm{Ag}(\mathrm{tcm})$ allows for different behaviour in the $a$ and $c$ directions, we find that both its compressibility and its thermal expansivity are essentially isotropic within the entire $(a, c)$ plane. Isotropy is important for NAC applications because it avoids composite warping and performance breakdown. ${ }^{2}$

That NAC is driven by the same layer-rippling mechanism as area-NTE is evident in the variation of the parameter $\theta$ with increasing pressure [Fig. 2(d)]. The volume reduction accommodated by rapid collapse of the interlayer spacing (note $K_{b} \gg$ $5 \mathrm{TPa}^{-1}$ ) evidently couples with a reduction in the degree of layer rippling: $\mathrm{d} \theta / \mathrm{d} p<0$. To substantiate further our interpretation of the NAC mechanism, we recast eqn (1) in terms of compressibilities; our refined values of $\theta$ give $K_{A}^{\text {calc }}=$ $-\theta \tan (\theta / 2) K_{\theta}=-26 \mathrm{TPa}^{-1}$. That the measured area compressibility $K_{(010)}=-7.5(8) \mathrm{TPa}^{-1}$ is somewhat smaller in magnitude reflects the expected $\sim 10 \mathrm{TPa}^{-1}$ contribution from (positive) bond compressibilities omitted from the geometric formalism of eqn (1), as discussed elsewhere for NLC materials. ${ }^{13}$ Indeed, that the $p / T$ correspondence inferred in Fig. 1(c) holds qualitatively for $\mathrm{Ag}(\mathrm{tcm})$ is immediately evident in the close relationship between the temperature- and pressure-induced lattice parameter and layer rippling parameter variations documented in Fig. 2.

In conclusion, we find that silver(I) tricyanomethanide exhibits NAC over the pressure range $0<p<0.615$ GPa with an essentially isotropic area compressibility of similar magnitude (but opposite sign) to that of established engineering materials. From a structural viewpoint, the phenomenon can be understood in terms of pressure-driven damping of layer 'rippling', acting to increase the layer cross-sectional area at larger hydrostatic pressures. Variable-pressure spectroscopic measurements may provide valuable additional insight into the subtle variations in bonding interactions that occur throughout this process. ${ }^{26}$ In favour of immediate application of $\mathrm{Ag}(\mathrm{tcm})$ in ultra-highprecision sensing devices are (i) the ease with which large single-crystal samples can be prepared under ambient conditions and using readily-available solvents, and (ii) its optical transparency. Moreover, the polarity of its crystal structure offers the possibility of designing multifunctional devices based on $\mathrm{Ag}(\mathrm{tcm})$ in which the interplay between responses to electric field, temperature, and pressure are exploited to produce piezoelectric and thermoelectric devices.

The authors gratefully acknowledge financial support from the E.P.S.R.C. (EP/G004528/2) and the E.R.C. (Grant Ref.: 279705), the provision of neutron beamtime on the PEARL instrument at ISIS, and the assistance of Dr Marek Jura (ISIS).

\section{References}

1 Z. G. Nicolaou and A. E. Motter, Nat. Mater., 2012, 11, 608-613.

2 R. H. Baughman, S. Stafström, C. Cui and S. O. Dantas, Science, 1998, 279, 1522-1524.

3 A. E. Aliev, J. Oh, M. E. Kozlov, A. A. Kuznetsov, S. Fang, A. F. Fonseca, R. Ovalle, M. D. Lima, M. H. Haque, Y. N. Gartstein, M. Zhang, A. A. Zakhidov and R. H. Baughman, Science, 2009, 323, 1575-1578.

4 R. J. Nelmes and A. Katrusiak, J. Phys. C: Solid State Phys., 1986, 19, L725-L730.

5 A. Sani, M. Hanfland and D. Levy, J. Phys.: Condens. Matter, 2002, 14, 10601-10604.

6 I. Loa, K. Syassen, R. K. Kremer, U. Schwarz and M. Hanfland, Phys. Rev. B, 1999, 60, R6945-R6948.

7 M. Y. Seyidov and R. A. Suleymanov, J. Appl. Phys., 2010, 108, 063540.

8 A. L. Goodwin, D. A. Keen and M. G. Tucker, Proc. Natl. Acad. Sci. U. S. A., 2008, 105, 18708-18713.

9 W. Li, M. R. Probert, M. Kosa, T. D. Bennett, A. Thirumurugan, R. P. Burwood, M. Parinello, J. A. K. Howard and A. K. Cheetham, J. Am. Chem. Soc., 2012, 134, 11940-11943.

10 H. J. Shepherd, T. Palamarciuc, P. Rosa, P. Guionneau, G. Molnár, J.-F. Létard and A. Bousseksou, Angew. Chem., Int. Ed., 2012, 51, 3910-3914.

11 A. B. Cairns, J. Catafesta, C. Levelut, J. Rouquette, A. van der Lee, L. Peters, A. L. Thompson, V. Dmitriev, J. Haines and A. L. Goodwin, Nat. Mater., 2013, 12, 212-216.

12 A. D. Fortes, E. Suard and K. S. Knight, Science, 2011, 331, 742-745.

13 J. M. Ogborn, I. E. Collings, S. A. Moggach, A. L. Thompson and A. L. Goodwin, Chem. Sci., 2012, 3, 3011-3017.

14 R. W. Munn, J. Phys. C: Solid State Phys., 1972, 5, 535-542.

15 A. B. Cairns, A. L. Thompson, M. G. Tucker, J. Haines and A. L. Goodwin, J. Am. Chem. Soc., 2012, 134, 4454-4456.

16 I. M. Lifshits, Zh. Eksp. Teor. Fiz., 1952, 22, 475-486.

17 M. J. Cliffe and A. L. Goodwin, J. Appl. Crystallogr., 2012, 45, 1321-1329.

18 J. Konnert and D. Britton, Inorg. Chem., 1966, 5, 1193-1196.

19 S. R. Batten, B. F. Hoskins and R. Robson, New J. Chem., 1998, 22, 173-175.

20 A. L. Goodwin, M. Calleja, M. J. Conterio, M. T. Dove, J. S. O. Evans, D. A. Keen, L. Peters and M. G. Tucker, Science, 2008, 319, 794-797.

21 R. E. Newnham, Properties of Materials, Oxford University Press, Oxford, 2005.

22 A. C. Bailey and B. Yates, J. Appl. Phys., 1970, 41, 5088-5091.

23 S. J. Hibble, A. M. Chippindale, A. H. Pohl and A. C. Hannon, Angew. Chem., Int. Ed., 2007, 46, 7116-7118.

24 P. Betteridge, J. Carruthers, R. Cooper, K. Prout and D. Watkin, J. Appl. Crystallogr., 2003, 36, 1487.

25 H. L. Zhang, S. Lu, M. P. J. Punkkinen, Q.-M. Hu, B. Johannson and L. Vitos, Phys. Rev. B, 2010, 82, 132409.

26 K. Kamali, C. Ravi, T. R. Ravindran, R. M. Sarguna, T. N. Sairam and G. Kaur, J. Phys. Chem. C, 2013, 117, 25704-25713. 